\title{
Incorporation of Prior Knowledge for Region of Change Imaging from Sparse Scan Data in Image-Guided Surgery
}

\author{
J. Lee ${ }^{\text {a }}$, J. W. Stayman ${ }^{\text {b }}$, Y. Otake ${ }^{\text {c }}$, S. Schafer ${ }^{\text {b }}$, W. Zbijewski ${ }^{\text {b }}$, A. J. Khanna ${ }^{\text {b,d }}$, J. L. Prince ${ }^{\text {a }}$, \\ and J. H. Siewerdsen ${ }^{\text {b,c }}$ \\ ${ }^{a}$ Department of Electrical and Computer Eng., Johns Hopkins University, Baltimore, MD, USA \\ ${ }^{\mathrm{b}}$ Department of Biomedical Engineering, Johns Hopkins University, Baltimore, MD, USA \\ ${ }^{\circ}$ Department of Computer Science, Johns Hopkins University, Baltimore, MD, USA \\ ${ }^{\mathrm{d}}$ Department of Orthopaedic Surgery, Johns Hopkins University, Baltimore, MD, USA
}

\begin{abstract}
This paper proposes to utilize a patient-specific prior to augment intraoperative sparse-scan data to accurately reconstruct the aspects of the region that have changed by a surgical procedure in image-guided surgeries. When anatomical changes are introduced by a surgical procedure, only a sparse set of $\mathrm{x}$-ray images are acquired, and the prior volume is registered to these data. Since all the information of the patient anatomy except for the surgical change is already known from the prior volume, we highlight only the change by creating difference images between the new scan and digitally reconstructed radiographs (DRR) computed from the registered prior volume. The region of change (RoC) is reconstructed from these sparse difference images by a penalized likelihood (PL) reconstruction method regularized by a compressed sensing penalty. When the surgical changes are local and relatively small, the RoC reconstruction involves only a small volume size and a small number of projections, allowing much faster computation and lower radiation dose than is needed to reconstruct the entire surgical volume. The reconstructed RoC merges with the prior volume to visualize an updated surgical field. We apply this novel approach to sacroplasty phantom data obtained from a conebeam CT (CBCT) test bench and vertebroplasty data with a fresh cadaver acquired from a C-arm CBCT system with a flat-panel detector (FPD).
\end{abstract}

Keywords: Prior knowledge, region of change imaging, sparse scan, image-guided surgery, C-arm, difference images, penalized likelihood reconstruction.

\section{INTRODUCTION}

Recent advancement of imaging technology and powerful computer and tracking systems combined with 3-D imaging techniques provide surgical navigation capabilities with a real-time visualization of the patient anatomy along with surgical instruments during the image-guided minimally invasive surgical procedure ${ }^{1}$. However, the relative position of any instrumentation to the patient anatomy varies as the surgical procedure progresses and surgical changes imparted to the patient anatomy cannot be accurately evaluated unless the surgical field is periodically updated during the surgery, which in turn significantly increases radiation dose and impedes current workflow. These limitations represent significant impediments to successful surgical outcomes.

In current image-guided surgery, pre- and/or intraoperative scans such as CT, MRI, and CBCT are routinely performed for diagnosis, surgical planning, and navigation systems ${ }^{2}$. Typically, intraoperative imaging for various image-guided surgeries such as orthopaedic, spinal, and vascular surgeries is carried out using C-arms that allow both real-time fluoroscopy and CBCT scans ${ }^{3}$. Recent advancement of FPD technology now brings next generation mobile Carm CBCT systems into clinic ${ }^{4}$. Especially, a surgical volume can be created at the beginning of the surgical process and an immediate registration of the instrument-tracking system to the surgical field can be made at the same patient position. However, radiation dose and workflow still remain unresolved challenges that prevent intraoperative $\mathrm{C}$-arm CBCT from being widely adopted in image-guided surgery. This is partly because each 3-D image acquisition requires a complete scan that imparts a full radiation dose, but does not utilize information from prior scans and presents the surgeon with a completely new 3 -D image every time.

This paper proposes a new strategy to achieve low-dose intraoperative 3-D imaging in image-guided surgery. The proposed method integrates prior information (simply called prior hereafter) of the patient anatomy into intraoperative $\mathrm{C}$-arm CBCT scans and involves minimal image acquisitions for accurate visualization of the region of surgical change. This new imaging approach can complement existing practice including conventions for intraoperative image acquisition

Medical Imaging 2012: Image-Guided Procedures, Robotic Interventions, and Modeling,

edited by David R. Holmes III, Kenneth H. Wong, Proc. of SPIE Vol. 8316, 831603

(C) 2012 SPIE · CCC code: 1605-7422/12/\$18 - doi: 10.1117/12.910850

Proc. of SPIE Vol. 8316 831603-1 
as well as use in navigation systems. The RoC reconstruction consists of the following steps: 1) registration of a patientspecific 3-D prior to intraoperative 2-D CBCT scans, 2) computation of difference images between the DRRs generated from the registered prior and the intraoperative $\mathrm{x}$-ray images, 3) reconstruction of the $\mathrm{RoC}$ from difference images using a PL reconstruction technique with a compressed-sensing (CS) type regularization ${ }^{5}$, and 4) fusion of the RoC reconstruction with the prior and visualization of the entire surgical field. Figure 1 shows the RoC reconstruction workflow. The proposed $\mathrm{RoC}$ reconstruction approach was validated on a simulated sacroplasty with a dry cadaveric bone and a vertebroplasty on a cadaveric torso, showing promising preliminary reconstruction results.

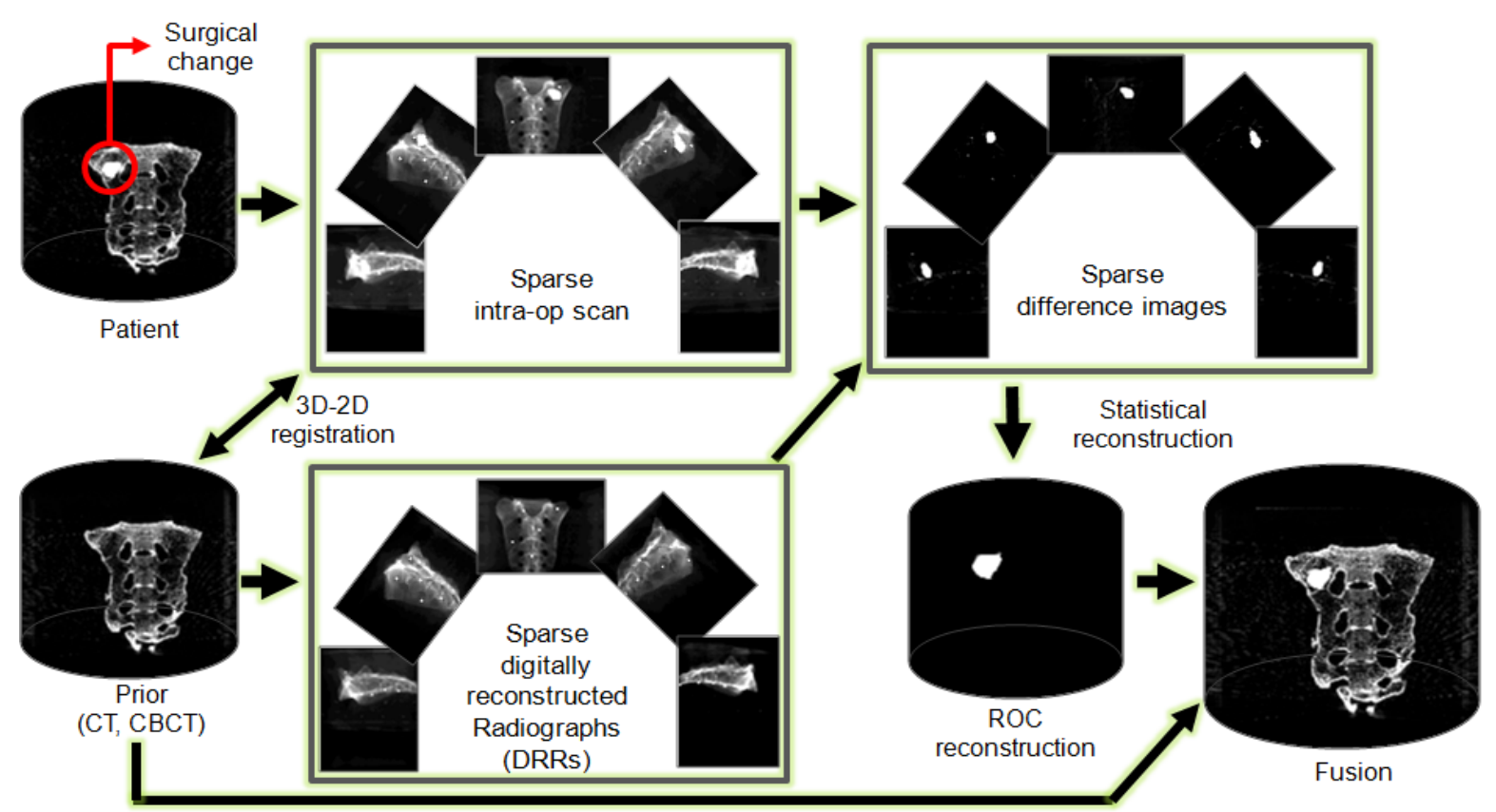

Figure 1. RoC reconstruction workflow. The surgical change illustrated is an injection of bone cement in a sacrum.

\section{METHODS}

In our approach, patient-specific prior knowledge is utilized to generate difference images that highlight surgical changes after being registered to intraoperative CBCT scans. Ideally, the difference images should show surgical changes only, and the region of the surgical change can be reconstructed from much smaller amount of data than is needed for reconstructing the entire surgical field. Therefore, our approach reconstructs RoC from a sparse set of projections rather than performing a complete $\mathrm{CBCT}$ scan in order to reduce radiation dose. The reconstructed RoC merges with the prior volume to present an updated 3-D surgical field.

When a sparse set of intraoperative x-ray images are obtained during the surgery, the 3-D prior volume is registered to a small subset (e.g., 4-6) of the obtained 2-D intraoperative projections. During the registration process, DRRs are repeatedly generated from the prior using a GPU and compared to the selected 2-D images. Data similarity between DRRs and the observed projections are computed by using a gradient difference similarity metric due to its robustness to slowly-varying soft tissue background and local data inconsistency introduced by surgical tools and implants ${ }^{6}$. The registration is optimized for pose by using downhill simplex method ${ }^{7}$. Once the prior is registered to the observed 2-D xray projections, we compute difference images by subtracting the DRRs from the intraoperative $\mathrm{x}$-ray images.

A 3-D RoC volume is reconstructed from the sparse set of difference images using a PL reconstruction method. In transmission tomography, measured $\mathrm{x}$-ray photons after being attenuated by an object under a monochromatic $\mathrm{x}$-ray assumption can be modeled as

$$
\bar{y}_{i}=I_{i 0} \exp \left(-[\mathbf{A} \mu]_{i}\right)
$$

where $I_{i 0}$ is the number of unattenuated photons, $\mu$ is the digitized linear attenuation map of the object, $\mathbf{A}$ is the system matrix, and $[\mathbf{A} \mu]_{i}$ is the line integral of the linear attenuation map $\mu$ measured at the detector element $i$. We assume that 
each measurement $y_{i}$ at the detector element $i$ is independent of the measurement in neighboring pixels and obeys a Poisson distribution with the expectation value of $\bar{y}_{i}$. Then, the joint probability of this measurement and the corresponding negative log-likelihood are given by

$$
\begin{gathered}
\boldsymbol{P}(y \mid \mu)=\prod_{i=1}^{N} \frac{\bar{y}_{i} y_{i}}{y_{i} !} \exp \left(-\bar{y}_{i}\right) \\
-L(y ; \mu)=\sum_{i=1}^{N}\left\{I_{i 0} \exp \left(-[\mathbf{A} \mu]_{i}\right)+y_{i}\left([\mathbf{A} \mu]_{i}\right)\right\}
\end{gathered}
$$

dropping the constant term. Direct minimization of (3) yields maximum likelihood (ML) estimate $\hat{\mu}$ which is very noisy. Alternatively, a penalized likelihood (PL) method regularizes the problem by applying roughness penalty to the objective function, thus mitigating artifacts and noise. In this approach, the following PL objective function modified from (3) is minimized:

$$
\hat{\mu}=\arg \min _{\mu}\{-L(y ; \mu)+\beta R(\mu)\},
$$

where $R(\mu)$ is the roughness penalty and $\beta$ controls the relative contribution of the likelihood and the penalty terms. In order to achieve CS-type regularization, we typically use a 1-norm to the spatial gradient $(\Psi)$ of the image estimate as our penalty, i.e., $R(\mu)=\|\Psi \mu\|_{1}$. It was reported that the PL reconstruction with CS-type penalty can provide improved reconstruction results compared to the total variation (TV)-based CS reconstruction ${ }^{5}$. Especially when the dose level is very low, TV-based CS reconstruction results in patchy images while mitigating streaking artifacts.

For the RoC reconstruction, we combine two sets of data; 1$)$ actual x-ray projections $\left(\bar{y}^{\text {intraop }}\right)$ obtained during the surgical procedure and 2) DRRs $\left(\bar{y}^{\text {prior }}\right)$ generated from the registered prior. The measured x-ray image $\bar{y}^{\text {intraop }}$ is created from the patient anatomy $\left(\mu^{\text {intraop }}\right)$ that contains the surgical change, but the DRR is generated from the prior volume $\left(\mu^{\text {prior }}\right)$ that does not have the information of the change. However, when we compute differences between these two data sets after logarithmic operation, the resulting difference image will be equivalent to the projection of the difference of the two volumes, i.e., $\mu^{\text {intraop }}-\mu^{\text {prior }}$ provided that the registration is accurate, and therefore can be described from (1) as

$$
\bar{y}_{i}^{\text {diff }}=I_{i 0} \exp \left(-\left[\mathbf{A}\left(\mu^{\text {intraop }}-\mu^{\text {prior }}\right)\right]_{i}\right)=I_{i 0} \exp \left(-\left[\mathbf{A} \mu^{\text {RoC }}\right]_{i}\right),
$$

which is the projection of the RoC. Although the difference images can be described as above, the likelihood has changed because this difference image computation involves a nonlinear subtraction of Poisson random variables. However, due to the complexity of this nonlinear subtraction, we presume that it is approximately Poisson in this paper (More accurate noise model is currently under investigation.) Under the Poisson assumption, $\mu^{R O C}$ can be estimated by minimizing the following objective function:

$$
\hat{\mu}^{R o C}=\arg \min _{\mu}\left\{-L\left(y^{\text {diff }} ; \mu\right)+\beta R(\mu)\right\} .
$$

Once the $\mathrm{RoC}$ is reconstructed, we can visualize the entire surgical volume by fusing the RoC reconstruction with the prior, i.e., $\hat{\mu}^{\text {intraop }}=\hat{\mu}^{R o C}+\mu^{\text {prior }}$. The PL estimation problem in (6) can be solved in various ways, and we typically used the ordered subset transmission tomography approach ${ }^{8}$ implemented on a GPU for a faster convergence.

To assess the image quality and accuracy of the RoC reconstruction, we compare it to a ground truth defined as a $\mathrm{CBCT}$ reconstruction computed from the complete data set (i.e., many projections acquired over a circular or semicircular arc reconstructed by FBP.) We used correlation coefficient (CC) and structural similarity index (SSIM) ${ }^{9}$ for quantitatively measuring the quality of the RoC reconstruction compared to the ground truth.

CC between two reconstructed volumes $\mathbf{x}=\left(x_{1}, \ldots, x_{\mathrm{n}}\right)$ and $\mathbf{y}=\left(y_{1}, \ldots, y_{\mathrm{n}}\right)$ are computed as

$$
\operatorname{CC}(\mathbf{x}, \mathbf{y})=\sum_{i=1}^{n} \frac{\left(x_{i}-\bar{x}\right)\left(y_{i}-\bar{y}\right)}{\sigma_{x} \sigma_{y}}
$$

where $\bar{x}$ and $\bar{y}$ are the mean values of $x_{i}$ 's and $y_{i}$ 's, and $\sigma_{x}$ and $\sigma_{y}$ are the standard deviations of $x_{i}$ 's and $y_{i}$ 's, respectively.

The SSIM consists of three similarity components; luminance $(l)$, contrast $(c)$, and structure $(s)$, that are computed as 


$$
\begin{gathered}
l(\mathbf{x}, \mathbf{y})=\frac{2 \bar{x} \bar{y}+C_{1}}{\bar{x}^{2}+\bar{y}^{2}+C_{1}} \\
c(\mathbf{x}, \mathbf{y})=\frac{2 \sigma_{x} \sigma_{y}+C_{2}}{\sigma_{x}^{2}+\sigma_{y}^{2}+C_{2}} \\
s(\mathbf{x}, \mathbf{y})=\frac{\sigma_{x y}+C_{3}}{\sigma_{x} \sigma_{y}+C_{3}}
\end{gathered}
$$

where $C_{1}, C_{2}$ and $C_{3}$ are constants included to avoid instability. We used the same $C_{1}, C_{2}$ and $C_{3}$ values in ref. 9. These three components are combined to form an overall SSIM as

$$
\operatorname{SSIM}(\mathbf{x}, \mathbf{y})=l(\mathbf{x}, \mathbf{y}) \times c(\mathbf{x}, \mathbf{y}) \times s(\mathbf{x}, \mathbf{y}) .
$$

\section{EXPERIMENTS AND RESULTS}

The $\mathrm{RoC}$ reconstruction was validated on two experiments; 1) sacroplasty on a dry cadaveric sacrum without soft tissue, and 2) vertebroplasty on a thoracic vertebra in a cadaveric torso. For the sacroplasty experiment, a CBCT test bench was used to acquire pre- and post-injection CBCT scans at $100 \mathrm{kVp}$ and $125 \mathrm{~mA}$ (500 mAs). Each image has the size of $1024 \times 768$ pixels with a pixel pitch of $0.388 \mathrm{~mm}$ after a $2 \times 2$ binning. A pre-injection CBCT volume of $400 \times 400 \times 400$ voxels with a voxel size of $0.5 \times 0.5 \times 0.5 \mathrm{~mm}^{3}$ was reconstructed from 360 projections scanned over $360^{\circ}$ arc and used as a prior. Bone cement was injected into the upper portion of the sacrum, and another full-sweep (360 projections over $360^{\circ}$ arc) CBCT scan was performed after the bone cement injection. A subset of 30 projections was extracted from the full scan data to simulate a sparse scan, which is equivalent to dose reduction by the factor of 12 . The prior was registered to post-injection $\mathrm{x}$-ray images using the 3D-2D rigid registration described in the previous section and difference images were generated. A RoC volume of $300 \times 300 \times 90$ voxels with a voxel size of $0.5 \times 0.5 \times 0.5 \mathrm{~mm}^{3}$ was reconstructed from these 30 difference images by the PL reconstruction method. The reconstructed RoC volume was compared to the ground truth that was computed by subtracting the pre-injection CBCT volume from the post-injection CBCT volume followed by a median filtering to remove quantum noise (Notice that the bone cement has uniform density.) CC and SSIM between the RoC reconstructions with different $\beta$ values and the ground truth are plotted in Figure 2, showing good agreement for all $\beta$ values. Since the dry sacrum did not have soft tissue, the 3D-2D registration was relatively easier and more accurate than a human subject with soft tissue. Therefore, the difference image computation was good enough to produce an accurate RoC reconstruction for any $\beta$ values. Example images of the computed difference image, the RoC reconstruction for $\beta=10^{7}$ (chosen from Figure 2) after 100 iterations, the fusion of the $\mathrm{RoC}$ reconstruction and the prior, the FBP reconstruction from the same set of 30 projections, and the post-injection CBCT computed from the complete data (360 projections) are displayed in Figure 3, showing significantly reduced artifact in $\mathrm{RoC}$ reconstruction compared to the FBP reconstruction.

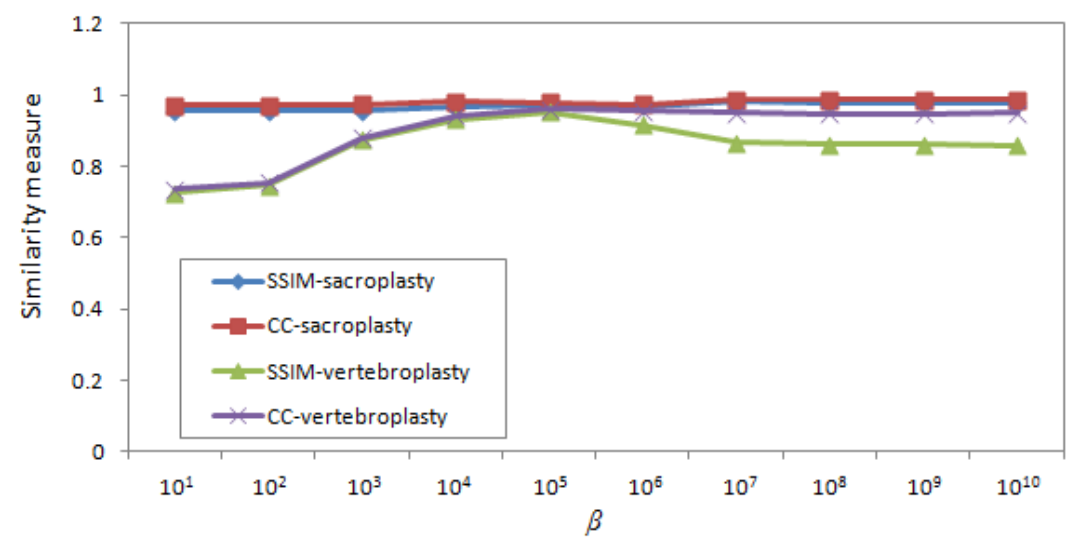

Figure 2. CC and SSIM plots for RoC reconstructions with different $\beta$ values. 


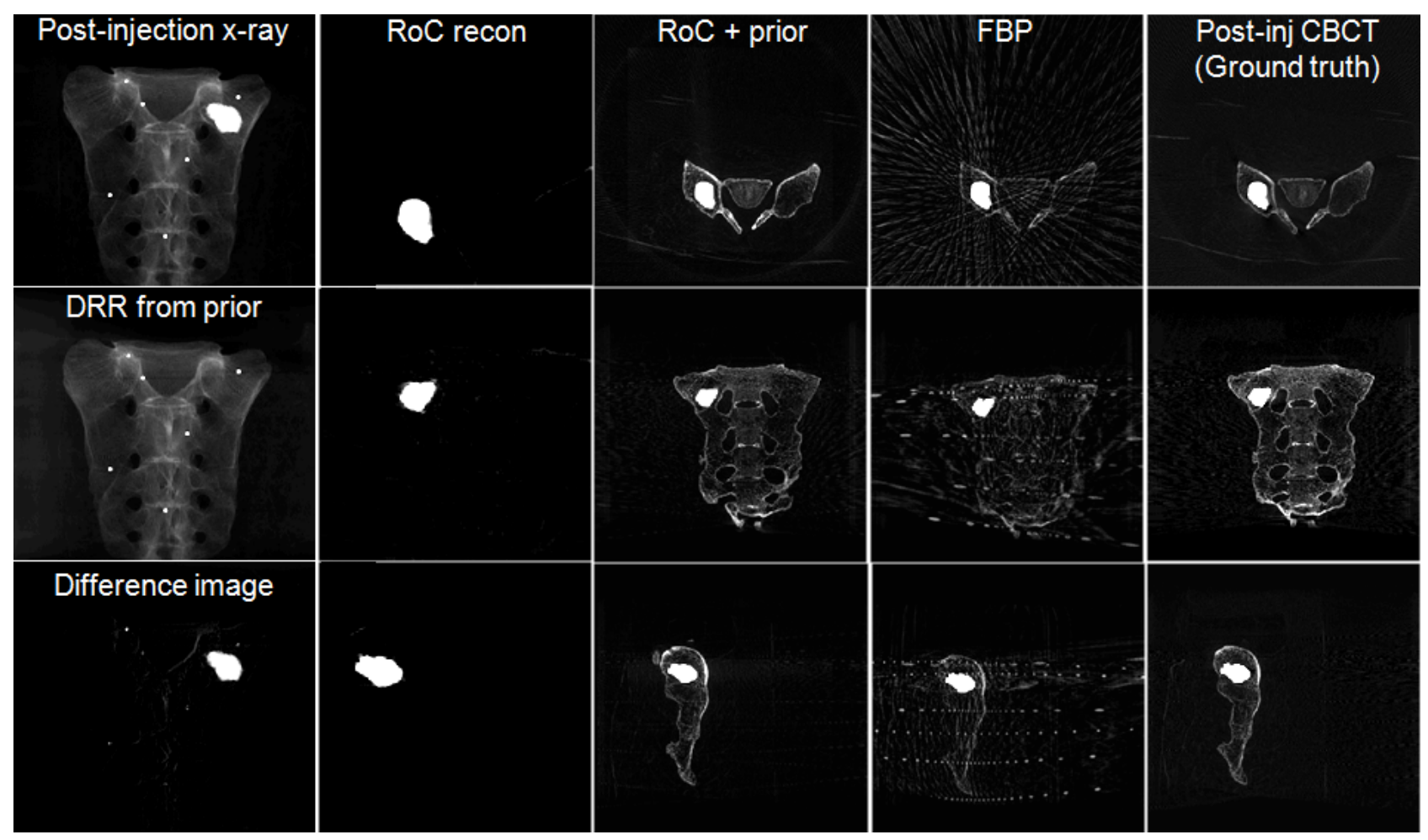

Figure 3. Sacroplasty phantom experiment. $\left(1^{\text {st }}\right.$ column $)$ Post-injection $x$-ray image, DRR created from the registered prior, and difference image. ( $2^{\text {nd }}$ column) RoC reconstruction (axial, coronal, sagittal) from 30 projections with $\beta=10^{7}$ after 100 iterations. ( $3^{\text {rd }}$ column) fusion of the RoC reconstruction with the prior (axial, coronal, sagittal). ( $4^{\text {th }}$ column) FBP reconstruction from 30 postinjection $\mathrm{x}$-ray images (axial, coronal, sagittal). ( $5^{\text {th }}$ column) Post-injection CBCT ground truth (axial, coronal, sagittal) computed from 360 projections.

In the vertebroplasty experiment, we used a FPD-based mobile C-arm (Siemens, Erlagen, Germany) for pre- and post-injection CBCT scans. A prior CBCT volume of $512 \times 512 \times 512$ voxels with a voxel size of $0.2930 \times 0.2930 \times 0.2930$ $\mathrm{mm}^{3}$ was reconstructed from 200 pre-injection $\mathrm{x}$-ray projections scanned over $178^{\circ}$ at the tube voltage of $120 \mathrm{kVp}$ and current of $2.3 \mathrm{~mA}(230 \mathrm{mAs})$. A surgeon carefully injected bone cement into a thoracic vertebra of a fresh cadaveric torso while minimizing its motion. After the bone cement injection, 200 post-injection x-ray images were acquired on the same $\mathrm{C}$-arm orbit. Difference images were computed by directly subtracting the pre-injection x-ray images from the post-injection x-ray images under the assumption that these two scans were registered (cadaver motion between these two scans was negligible) and the $\mathrm{C}$-arm image acquisition was reproducible, yielding reasonable difference images as shown in Figure 4. Among 200 difference images, we uniformly sampled a subset of 40 projections among 200 images to simulate a sparse intraoperative scan, which is equivalent to dose reduction by the factor of 5 . A RoC volume of $250 \times 250 \times 250$ voxels with a voxel size of $0.2930 \times 0.2930 \times 0.2930 \mathrm{~mm}^{3}$ was reconstructed by the PL reconstruction method, and compared to the ground truth that was computed by subtracting the pre-injection CBCT from the postinjection $\mathrm{CBCT}$ followed by a median filtering to remove quantum noise. CC and SSIM between the RoC reconstructions with different $\beta$ values and the ground truth are plotted in Figure 2. Figure 4 shows example images of the computed difference image, the RoC reconstruction result for $\beta=10^{5}$ (chosen from Figure 2) after 100 iterations, the fusion of $\mathrm{RoC}$ with the prior, the FBP reconstruction from the same 40 post-injection $\mathrm{x}$-ray images, and the postinjection CBCT computed from the complete data (200 projections). The artifacts in the FBP reconstruction are much severe than the sacroplasty case due to the soft tissue and data truncation in this case, but these reconstruction artifacts are removed in the $\mathrm{RoC}$ reconstruction. 


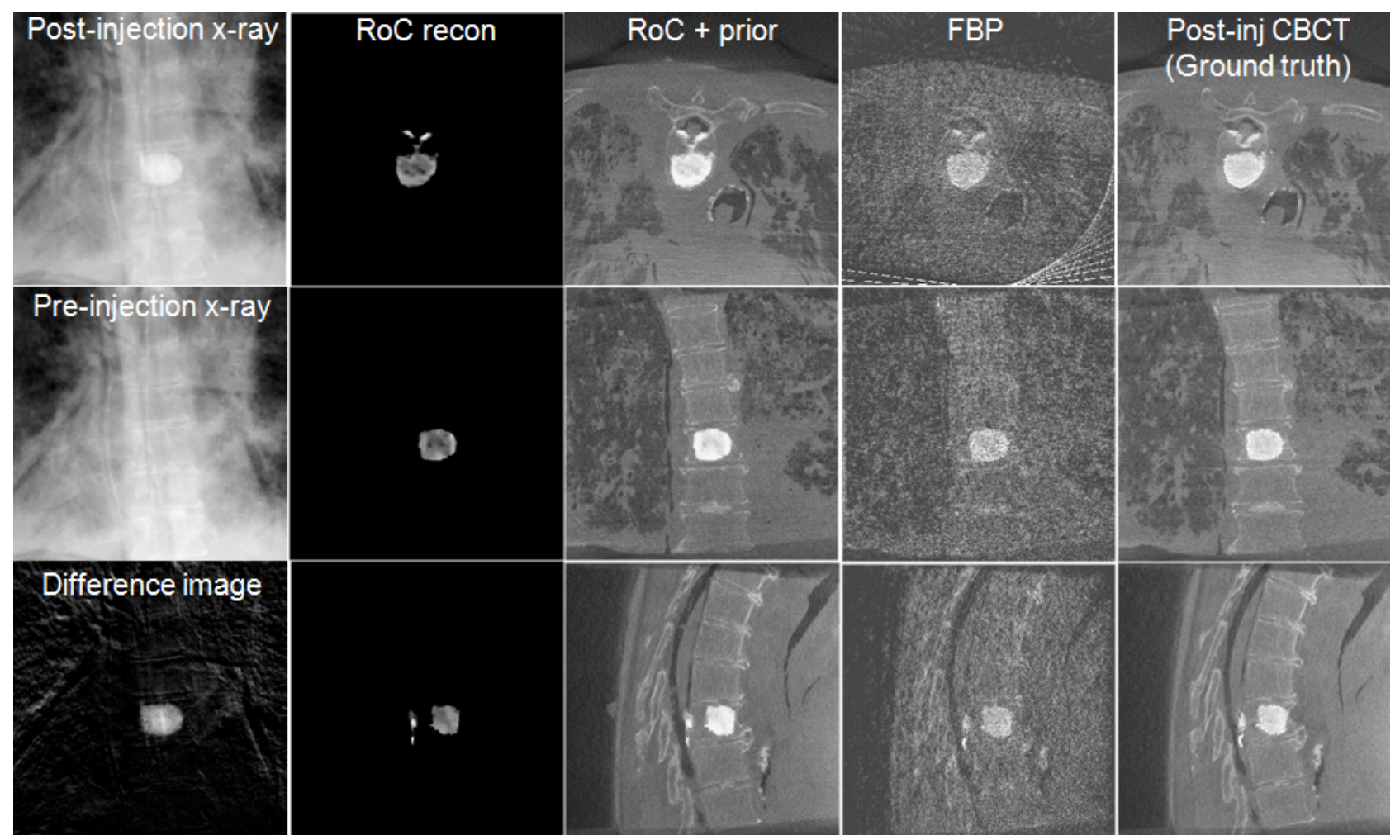

Figure 4. Vertebroplasty cadaver experiment. ( $1^{\text {st }}$ column) Post-injection x-ray image, pre-injection x-ray image, and difference image. ( $2^{\text {nd }}$ column) RoC reconstruction (axial, coronal, sagittal) from 40 projections with $\beta=10^{5}$ after 100 iterations. ( $3^{\text {rd }}$ column) fusion of the RoC reconstruction with the prior (axial, coronal, sagittal). $\left(4^{\text {th }}\right.$ column) FBP reconstruction (axial, coronal, sagittal) from 40 post-injection $x$-ray images. ( $5^{\text {th }}$ column) Post-injection CBCT ground truth (axial, coronal, sagittal) computed from 200 projections.

\section{CONCLUSIONS}

The key benefit surgeons expect from the image-guidance in minimally invasive surgery is an accurate visual feedback about the surgical change occurred in the patient. Under this idea, we proposed a method to fully utilize existing patientspecific prior knowledge from the previous scans to complement sparse-scan intraoperative data by generating difference projections that highlight only the surgical changes. High-quality RoC reconstruction is computed from these sparse set of difference projections by using a PL reconstruction with CS-type penalty. The reconstructed RoC merges with the prior to visualize a complete surgical field. The concept of RoC imaging, rather than refreshing the entire surgical field with completely new 3-D images, is an innovative paradigm shift from conventional CT reconstructions, and will change the way how image-guided minimally invasive surgeries are carried out, thus removing the primary barriers to broader acceptance of image-guided minimally invasive surgery and facilitating improved surgical procedures and outcomes with lower radiation dose.

\section{REFERENCES}

[1] Cleary, K. and Peters, T. M., "Image-guided interventions: technology review and clinical applications," Annu. Rev. Biomed. Eng., 12, 119-142 (2010).

[2] Schoenfeld, A. J., Bono, C. M., McGuire, K. J., Warholic, N., Harris, M. B., "Computed Tomography Alone Versus Computed Tomography and Magnetic Resonance Imaging in the Identification of Occult Injuries to the Cervical Spine: A Meta-Analysis," J. Trauma-Inj Inf Critical Care, 68 (1), 109-113 (2010).

[3] Nolte, L. -P., Slomczykowski, M. A., Berlemann, U., Strauss, M. J., Hofstetter, R., Schlenzka, D., Laine, T., and Lund, T., "A new approach to computer-aided spine surgery: fluoroscopy-based surgical navigation," Eur. Spine. J., 9, S78-S88 (2000). 
[4] Siewerdsen, J. H., Daly, M. J., Chan, H., Nithiananthan, S., Hamming, N., Brock, K. K., and Irish, J. C., "Highperformance intraoperative cone-beam $\mathrm{CT}$ on a mobile $\mathrm{C}$-arm: an integrated system for guidance of head and neck surgery," Proc. SPIE Med. Imag., 7261, 72610J (2009).

[5] Tang, J., Nett, B. E., Chen, G. -H., "Performance comparison between total variation (TV)-based compressed sensing and statistical iterative reconstruction algorithms," Phys. Med. Biol., 54 (19), 5781-5804 (2009).

[6] Penney, G. P., Weese, J., Little, J. A., Desmedt, P., Hill, D. L. G., Hawkes, D. J., "A comparison of similarity measures for use in 2-D-3-D medical image registration," IEEE Trans. Med. Imag. 17 (4), 586-595 (1998).

[7] Nelder, J. A. and Mead, R., "A simplex method for function minimization," Computer J., 7, 308-313 (1965).

[8] Erdogan, H. and Fessler, J. A., "Ordered subsets algorithms for transmission tomography," Phys. Med. Biol. 44, 2835-2851 (1999).

[9] Wang, Z., Bovik, A. C., Sheikh, H. R., Simoncelli, E. P., "Image Quality Assessment: From Error Visibility to Structural Similarity," IEEE Trans. Imag. Proc., 13 (4), 600-612 (2004). 